\title{
High-Flow Nasal Cannula using a Ventilator or Non- Invasive Ventilation for Covid-19-Associated Acute Respiratory Failure
}

\author{
Miyuki Okuda*, Toshiya Maekura and Yuto Kido \\ Osaka Anti-tuberculosis Association Osaka Fukujuji Hospital, Japan
}

*Corresponding author: Miyuki Okuda, Osaka Anti-tuberculosis Association Osaka Fukujuji Hospital, 3-10 Uchiagetakatsuka, Neyagawa, Osaka 572-0850, Japan.

To Cite This Article: Miyuki Okuda, Toshiya Maekura and Yuto Kido. High-Flow Nasal Cannula using a Ventilator or Non-Invasive Ventilation for Covid-19-Associated Acute Respiratory Failure. Am J Biomed Sci \& Res. 2021 - 14(1). AJBSR.MS.ID.001952.

DOI: 10.34297/AJBSR.2021.14.001952.

Received: 嗹 August 24, 2021; Published: 㘹 August 30, 2021

Keywords: High-flow Nasal Cannula; Covid-19; Acute Respiratory Failure

\section{Introduction}

High-flow nasal cannula (HFNC) for acute respiratory failure significantly reduces overall mortality, intubation requirements and nosocomial pneumonia, and improves patient comfort when compared with noninvasive ventilation (NIV) and conventional oxygen therapy (COT) [1]. The severe acute respiratory syndrome coronavirus 2 (SARS-CoV-2) disease 2019 (COVID-19) pandemic is still ongoing [2]. A randomized trial of HFNC in patients with severe COVID-19 reported significantly lower respiratory rates and improved arterial/inspired oxygen (P/F) ratios at 6 hours, and a reduction in ICU stay, with HFNC versus COT $[3,4]$. The Surviving Sepsis Campaign Guidelines also recommend HFNC as oxygen therapy over COT and NIV [5].

However, HFNC is associated with respiratory aerosol generation, although the amount and concentration of aerosol produced at a distance of $10 \mathrm{~cm}$ from the mouth by HFNC, nasal cannula, oxygen mask, and normal breathing are reported to be similar [6].

The WHO recommends the use of surgical masks to prevent infection transmission. Reportedly, wearing a mask while receiving HFNC can reduce the number of droplets dispersed in the environment [7]. Furthermore, wearing a surgical mask over an HFNC device can reduce COVID-19-related hypoxemia, in terms of $\mathrm{PaO}_{2}$ (from $59( \pm 6)$ to $79 \mathrm{mmHg}( \pm 16), \mathrm{p}<0.001$ ) and $\mathrm{PaO}_{2} /$ $\mathrm{FiO}_{2}$ (from $83( \pm 22$ ) to $111( \pm 38), \mathrm{p}<0.001$ ) [8]. Therefore, it is recommended that patients receiving HFNC should wear a surgical mask to prevent infection transmission by aerosols and improve respiratory failure.

Although HFNC is useful for acute respiratory failure caused by COVID-19, the required equipment might not be readily available. In such cases, a ventilator with a constant pressure control mode or NIV/CPAP equipment can be used for HFNC [9]. The principle of pressure-controlled HFNC is described below.

\section{a. Principle}

When fluid flows in a pipe, the law of conservation of mass describes the correlation between flow rate $(Q)$, density $(\rho)$, crosssectional area (S) and velocity (v) as:

\section{$Q=\rho S \_1 v_{-} 1=\rho S_{-} \_2 v_{-} 2 \quad$ (1) (Equation of continuity)}

According to the law of conservation of energy, pressure (P), height $(\mathrm{h})$, and gravitational acceleration $(\mathrm{g})$ are related by the formula: 
$\mathrm{P}_{-} 1+\rho g h+1 / 2 \quad \rho v_{-} 1^{\wedge} 2=\mathrm{P}_{-} 2+\rho g h+1 / 2 \quad \rho v_{-} 2^{\wedge} 2$ (Bernoulli's theorem)

Substituting equation (1) in equation (2), we get:

$$
Q=\sqrt{ }\left(2 \rho\left(P_{-} 1-P_{-} 2\right)\right) \cdot\left(S_{-} 1 \cdot S_{-} 2\right) / \sqrt{ }\left(S_{-} 1^{\wedge} 2-S_{-} 2^{\wedge} 2\right)
$$

Therefore, the flow rate $\mathrm{Q}$ is determined by $\mathrm{P}_{-}$1-P_2 (pressure gradient $\Delta \mathrm{P}$ ) and cross-sectional areas $\mathrm{S} \_1$ and $\mathrm{S} \_2$. If we consider S_1 as the diameter of the circuit and $\mathrm{S}_{-} 2$ as the diameter of the cannula, the flow rate will remain constant when the pressure is kept constant while using the same circuit and cannula.

According to this principle, a pressure-controlled device is a device that produces a constant flow rate using a function to control pressure at a constant level, such as CPAP. The flow rate is almost the same as the leakage volume.

We performed pressure-controlled HFNC using a ViVO50 ventilator (Breas Medical AB, Mölnlycke, Sweden) and flowcontrolled oxygen device (AIRVO 2; Fisher \& Paykel Healthcare, Auckland, New Zealand), and flow-controlled ventilator (Steady Air; Atom Medical, Tokyo, Japan) In this study, we investigated the physiological effects of use of pressure-controlled HFNC flowcontrolled AIRVO 2, and Steady Air in healthy subjects [10]. With all methods, ventilatory volume increased, transcutaneous carbon dioxide partial pressure and respiratory frequency decreased, and inspiratory/expiratory esophageal pressure decreased. In addition, no significant differences between the three methods were observed in any items. Therefore, we believe that HFNC can be performed using a blower system with the capability of controlling pressure at a constant level, such as CPAP. If intubation can be avoided by HFNC, the utilization rate of ICUs, which is currently, globally, very high, might decrease, and the number of lives that can be saved might increase. Furthermore, if pressure-controlled HFNC using a ventilator or CPAP/NIV is possible, the number of HFNCs will increase, reducing the overall cost of the devices, and the use of a ventilator or CPAP/NIV in the same circuit will consequently have a lower treatment cost. However, it is important to confirm maintenance of adequate oxygenation at the recommended saturation level of $92-96 \%$ when administering HFNC with these devices.
In conclusion, we would like to suggest that HFNC with a ventilator or NIV/CPAP device might be useful in the management of acute respiratory failure due to COVID-19.

\section{References}

1. Baldomero AK, Melzer AC, Greer N, Brittany N Majeski, Roderick Macdonald, et al. (2021) Effectiveness and harms of High-flow nasal cannula for acute respiratory failure: An evidence report for a clinical guideline from the American College of Physicians. Ann Intern Med 10(2): e034956.

2. Zhou F, Yu T, Du R, Guohui Fan, Ying Liu et al. (2020) Clinical course and risk factors for mortality of adult inpatients with COVID-19 in Wuhan, China: a retrospective cohort study. Lancet 395(10229):1054-1062.

3. Teng XB, Shen Y, Han MF, Gang Yang, Lei Zha, et al. (2021) The value of high-flow nasal cannula oxygen therapy in treating novel coronavirus pneumonia. Eur J Clin Invest 51(3):e13435.

4. Mellado-Artigas R, Ferreyro BL, Angriman F, María Hernández-Sanz, Egoitz Arruti, et al. (2021) High-flow nasal cannula in patients with COVID-19-associated acute respiratory failure. Crit Care 25(1): 58.

5. Alhazzani W, Møller MH, Arabi YM, Mark Loeb, Michelle Ng Gong, et al. (2020) Surviving Sepsis Campaign: guidelines on the management of critically ill adults with Coronavirus Disease 2019 (COVID-19). Intensive Care Med 46(5): 854-887.

6. Iwashyna TJ, Boehman A, Capecelatro J, Amy M Cohn, James M Cooke et al. (2020) Variation in aerosol production across oxygen delivery devices in spontaneously breathing human subjects. medRxiv 1-11.

7. Leonard S, Strasser W, Whittle JS, Leonithas I Volakis, Ronald J DeBellis, et al. (2020) Reducing aerosol dispersion by High flow therapy in COVID-19: High resolution computational fluid dynamics simulations of particle behavior during high velocity nasal insufflation with a simple surgical mask. Am Coll Emerg Physicians Open 1(4): 578-591.

8. Montiel V, Robert A, Robert A, Anas Nabaoui, Tourneux Marie, et al. (2020) Surgical mask on top of high-flow nasal cannula improves oxygenation in critically ill COVID-19 patients with hypoxemic respiratory failure. Ann Intensive Care 10(1): 125.

9. Okuda M, Kashio M, Tanaka N, Tomoshige Matsumoto, Sumiko Ishihara, et al. (2014) Nasal high-flow oxygen therapy system for improving sleep-related hypoventilation in chronic obstructive pulmonary disease: a case report. J Med Case Rep 8: 341.

10. Okuda M, Tanaka N, Naito K, Takao Kumada, Koji Fukuda, et al. (2017) Evaluation by various methods of the physiological mechanism of a high-flow nasal cannula (HFNC) in healthy volunteers. BMJ Open Respir Res 4(1): e000200. 